\title{
Development and Pilot Test of a Physician-Focused Cancer Clinical Trials Communication Training Intervention
}

\section{Susan Eggly ( $\sim$ egglys@karmanos.org )}

Wayne State University School of Medicine https://orcid.org/0000-0002-8137-6098

Mark Manning

Wayne State University School of Medicine

Nicole Senft

Wayne State University School of Medicine

Tanina Foster Moore

Wayne State University School of Medicine

Terrance L. Albrecht

Wayne State University School of Medicine

Louis A. Penner

Wayne State University School of Medicine

Elisabeth Heath

Wayne State University School of Medicine

Michael A. Carducci

Johns Hopkins Medicine Sidney Kimmel Comprehensive Cancer Center

Dina G. Lansey

Johns Hopkins Medicine Sidney Kimmel Comprehensive Cancer Center

Lauren M. Hamel

Wayne State University School of Medicine

Research

Keywords: patient-provider communication, healthcare disparities, clinical trials, oncology, communication training

Posted Date: March 8th, 2021

DOI: https://doi.org/10.21203/rs.3.rs-281271/v1

License: (c) (1) This work is licensed under a Creative Commons Attribution 4.0 International License.

Read Full License 
Version of Record: A version of this preprint was published at PEC Innovation on December 1st, 2021. See the published version at https://doi.org/10.1016/j.pecinn.2021.100012. 


\section{Abstract}

\section{Purpose}

Clinical trials are critical to advancing cancer treatments, yet their impact is hindered by low accrual rates, especially among minorities. One barrier is the quality of physician communication during clinical interactions in which trials are discussed. We describe the development and pilot test of a physicianfocused, web-based training module designed as an intervention to improve physician communication related to discussing clinical trials in a diverse cancer patient population. The purpose of the pilot test was to assess acceptability and preliminary effectiveness in improving physician beliefs, attitudes, knowledge, and comfort related to discussing cancer clinical trials with patients and families in a diverse patient population.

Method

A team of researchers, clinicians, and community members developed the training module based on current communication research and analysis of an archive of video-recorded patient-oncologist interactions. The module included a brief didactic video explaining patient-centered communication strategies for discussing trials, and re-enactments of selected video clips from the archive to provide illustrations and guide opportunities for reflection. For the pilot test, the module was provided to physician participants in the Partnering Around Cancer Clinical Trials (PACCT) trial at two NCl-designated comprehensive cancer centers. Pre-post measures included assessments of change in beliefs, behavioral attitudes, knowledge and comfort related to discussing trials; measures also assessed evaluations of the module.

\section{Results}

Nineteen physicians participated in the pilot test of the module. Most were very experienced in discussing trials. Pre-post assessments indicated mixed findings about beliefs, marginal improvement in attitudes, and a significant improvement in knowledge, but no change in comfort discussing trials. Feedback on the module was favorable.

Conclusions

This pilot test of a stakeholder-developed physician communication training module demonstrated its acceptability and effectiveness, even in this highly-experienced physician sample. Future research is needed to determine its effectiveness on communication in clinical settings.

Trial Registration Number NCT02906241

\section{Introduction}


Cancer clinical trials are essential for testing the safety and efficacy of promising treatments and translating new knowledge into tangible benefits for patients; they also represent state-of-the art treatment for individuals with cancer. However, only a small proportion of people with cancer ever enroll in a trial. Under-enrollment is a greater problem among minorities, particularly African Americans, suggesting a healthcare disparity and limiting the generalizability of findings across populations.[1, 2]

Barriers to clinical trial enrollment exist at every level.[3, 4] Still, most efforts to increase enrollment focus on patients' and community members' awareness, knowledge, and attitudes related to clinical trials.[5-8] Other efforts focus on system-level factors, such as increasing the number of available trials, providing resources to under-resourced medical institutions, and broadening eligibility criteria.[9] However, one barrier that has received relatively little attention is physician communication during clinical interactions with patients who are potentially eligible for an available trial.

Studies show that most patients, if eligible for an available trial, agree to participate when their physician offers them the opportunity and explicitly recommends they consider participating using clear, patientcentered communication.[10-12] Unfortunately, oncologists infrequently discuss trials with their eligible patients $[13,10]$ due to a lack of awareness of available trials, negative attitudes about the time and resources required to discuss and enroll patients in trials, negative beliefs about the scientific value of trials, and perceptions that their patients are not interested in participating.[4] When oncologists do discuss trials with eligible patients, they often use confusing or misleading language, such as omitting key aspects of the trial (e.g., the purpose of the study), exaggerating benefits, and minimizing risks.[14] Also, the quality of clinical communication (including communication about clinical trials) has been shown to be poorer during interactions with African-American patients than in those with White patients. [15] Our own observational research has demonstrated that when African Americans are invited to participate in a trial, they receive less information about the trial than White patients.[16]

Existing interventions to improve awareness, attitudes, knowledge, and communication skills related to patient-physician discussions about trials[17] have several limitations. First, most interventions focus on the communication skills of patients, rather than physicians.[18] Second, physician communication training in the oncology setting often fails to include content specific to discussing clinical trials or about discussing trials with racially/ethnically diverse patient populations.[19, 20] Third, physician communication training generally utilizes simulated scenarios rather than naturally-occurring clinical interactions to illustrate skills and facilitate skills practice. Although simulated scenarios can be helpful for illustrating a specific teaching objective, medical trainees often view simulated scenarios as contrived or inauthentic,[21] a view likely to be even stronger among experienced clinicians. Video recordings of naturally-occurring trial discussions collected for research purposes contain rich examples of effective and ineffective strategies oncologists use to manage the challenges of clinical encounters. De-identified, professional re-enactments of these interactions offer naturalistic opportunities for learning and reflection that can be disseminated for training. 
We describe the development and pilot-testing of a web-based physician training module using professional re-enactments of actual clinical interactions. The module was designed as an intervention to improve physicians' knowledge, beliefs, and attitudes related to discussing cancer clinical trials with patients and their families in a diverse patient population. The pilot test was designed to assess the extent to which the training module improved physicians' beliefs, knowledge, attitudes, and comfort related to discussing clinical trials with patients and families, as well as to obtain their general feedback on the acceptability of the training module. Findings can inform a future randomized controlled trial to assess the extent to which the intervention improves trial-related communication in clinical settings.

\section{Development of the Training Module}

The module was developed by the investigators and stakeholders, including oncologists, survivors, and community members. The module was designed to be accessed via a website, and included a brief didactic video followed by re-enacted clinical interactions in which clinical trials were discussed.

Didactic video: The professionally-produced didactic video included physicians and health communication scientists from two National Cancer Institute-designated comprehensive cancer centers (John Hopkins Sidney Kimmel Cancer Center [JHU] and Wayne State University/Karmanos Cancer Institute [WSU-KCI]). These professionals described the importance of recruiting a diverse population of patients to cancer clinical trials using high-quality, patient-centered communication involving the exchange of informational and relational messages.[10] Informational communication was described as discussing information that patients need to make an informed decision about participating in a specific trial, including the purpose of the trial; risks (including side effects); benefits to the individual and/or society; alternatives to participating in the trial; and that participation in the trial is voluntary.[22] Relational communication was described as using strategies for using clear, effective, patient-centered communication, such as building trust, encouraging questions, using lay language, including family members, making clear transitions between topics, and using "ask-tell-ask" and "teach-back".[23, 24] (The didactic video received a Silver and a Gold Telly Award in 2020, an award that honors excellence in video and television across all screens.)

Re-enactments: Professional actors re-enacted video-recorded patient-oncologist interactions collected as part of a prior study investigating patient-oncologist communication about clinical trials.[10] The reenactments illustrated points made in the didactic video and gave physicians an opportunity to observe and reflect on clinical communication that naturally occurred in clinical settings that may be similar to their own practices. To select video segments to re-enact, we identified videos that included an explicit trial offer $(n=39)$. Faces in the videos were blurred to protect confidentiality. A panel of stakeholders (three clinical oncologists, two communication scientists, and three community members) identified portions of the interactions they deemed important for training oncologists to communicate effectively about trials. Based on the expert panel's recommendations, we selected 13 video segments which clearly demonstrated an oncologist (1) discussing at least one key elements of consent and (2) using particularly high- or low-quality relational communication. These segments were transcribed verbatim to 
form scripts for the re-enactments. A professional production company produced the re-enactments with actors selected for their skill and for the extent to which their appearance was similar to the patients, family members, and physicians in the original interactions. To increase authenticity, recording of the reenactments took place in one of the outpatient clinics where the original interactions had occurred and were recorded using similar equipment.

Following production, stakeholders (9 oncologists and 10 cancer survivors) viewed the re-enactments to judge the extent to which they were informative, realistic, and valuable for training oncologists, and the extent to which physicians in the re-enactments used lay language, clear and easy explanations, were informative and thorough, and seemed to care about the patients. Stakeholder judgements were favorable on all items and thus all of the re-enactments were included in the training module along with open-ended questions to encourage physicians to reflect on their own communication skills.

\section{Pilot-Testing of the Training Module}

\section{Setting, Participants, and Procedures}

The goal of the pilot test was to assess the extent to which the training module improved physicians' beliefs, attitudes, knowledge, and comfort related to discussing clinical trials with patients and families, as well as to obtain their general feedback on the acceptability of the training module. Physicians participating in a larger study designed to improve clinical trial communication and accrual rates among Black and White men with prostate cancer were eligible and invited to complete the module.[25] The larger study was conducted at two National Cancer-Institute-designated comprehensive cancer centers, one in Detroit, Michigan (WSU/KCl) and the other in Baltimore, Maryland (JHU). Briefly, for the larger study, patient participants received a patient-focused intervention via a between-subjects design (Phases 1 and 2), and physician participants received the training module as part of a physician-focused intervention two years into the study via a within-subjects design (Phase 2). From February-November, 2019, physician participants were provided a link to access the module online and given two weeks to complete it. All procedures were approved by the institutional review boards at both hospitals and universities.

Assessments: Unless otherwise indicated, participants completed items at the beginning and/or end of the module to assess the following:

Sociodemographic and professional characteristics included clinical specialty, years in practice, practice site, gender, race/ethnicity, and age.

Greatest perceived barrier to clinical trial offers was indicated with choice of one among the following options: lack of available trials, lack of institutional support, lack of awareness of available trials, or belief that patients prefer not to discuss trials.

Beliefs about clinical trials were assessed with a 13-item measure[26] of beliefs about three dimensions of clinical trials: patient care (e.g., "Clinical trials improve patient care in general");" profession (e.g., 
"Clinical trials are an appropriate use of resources"; and available resources (e.g., "Information about clinical trials is easily available to me"). Responses ranged from $1=$ strongly disagree to $5=$ strongly agree.

Behavioral attitudes towards clinical trials were assessed with 6 semantic differential items (i.e., unpleasant - pleasant; harmful - beneficial; not enjoyable - enjoyable; worthless - valuable; not preferable - preferable; useless - useful) beginning with the stem: "Offering a clinical trial to my eligible patients with prostate cancer would be..." Responses were rated on a 5-point scale.

Knowledge of trial-related information and communication skills that should be used during clinical trials discussions with patients was assessed with 11 items in multiple choice or true-false formats. Some examples were "Which of the following best describes the "teach-back" method of communicating with patients?" and "As long as the purpose section of a clinical trial's informed consent form is full and complete, there is no reason to tell the patient that the trial is research."

Comfort with discussing clinical trials was assessed with 1 item ("I am comfortable discussing clinical trials with my patients,") with responses ranging from $1=$ strongly disagree to $5=$ strongly agree.

Course Evaluation: Participants were asked to evaluate three aspects of the course. The didactic video evaluation, presented following the video, included 6 items, (e.g., "The contents of this video will be valuable in discussing clinical trials with my patients and their families"). The re-enactments evaluation, presented following the re-enactments, used the same six items used to evaluate the didactic video plus two additional items, ("There was too much new information" and "I enjoyed the (reenactment) videos"). All responses ranged from $1=$ strongly disagree to $5=$ strongly agree. Finally, at the end of the module, 3 questions assessed the extent to which the course achieved its objectives (e.g., "Learners will be able to identify strategies for discussing trials with patients and families"; responses ranged from 1 [not well at all] to 5 [very well]), and one question assessed whether physicians would make changes in their practice based on what they learned in the course (yes/no). Participants were also asked to provide general comments and/or suggestions for future activities.

\section{Results}

Twenty-one oncologists were eligible and invited to take the training module as part of their participation in a larger study; two declined. A description of the socio-demographic and professional characteristics of the 19 participants is provided in Table 1. In the past year one participant (5.3\%) had offered a clinical trial to an eligible patient 1-2 times; 2 (10.5\%) had made an offer $3-5$ times; and 16 (84.2\%) had made an offer 6 or more times, indicating these physicians had a high level of experience in discussing trials with patients and families. Participants' greatest perceived barrier to offering trials was lack of time $(n=8$, $42.1 \%)$; followed by no trials available $(n=3,15.8 \%)$; lack of awareness of available trials $(n=3,15.8 \%)$; lack of institutional support $(n=1,5.3 \%)$; and other (overwhelming regulatory work $(n=1)$, funding constraints $(n=1)$, patients not eligible $(n=1)$, or travel distance difficult for patients $(n=1))$. No participant selected the response indicating the perception that patients preferred not to discuss trials. 
Table 2 presents correlations among the 3 dimensions of clinical trial beliefs, behavioral attitudes, knowledge, and comfort discussing clinical trials before and after completing the training module. Preand post-training means are presented in Table 3. We used repeated measures ANOVA with within-subject effects only to examine whether post-training scores differed significantly from pre-training scores. Results (Table 3) indicated a significant improvement in clinical trial beliefs about patient care $\left(F_{1,18}=\right.$ 9.50, $p=.006, \eta^{2}=.35$ ), but no significant change in clinical trial beliefs related to the profession or to available resources. Behavioral attitudes marginally improved $\left(F_{1,18}=3.30, p=.09, \eta^{2}=.16\right)$, and knowledge of trial-related information and communication significantly improved $\left(F_{1,18}=12.64, p=.002\right.$, $\left.\eta^{2}=.41\right)$. There was no significant change in physicians' comfort discussing trials.

Participant evaluations of the course were generally favorable. Mean evaluations (on a 5-point scale) were as follows: Didactic videos $(M=3.89, S D=.52)$, re-enactments $(M=3.63, S D=.557)$; achieved learning objectives $(M=4.35, S D=.65)$. Most participants $(68.4 \%)$ said they would make changes in their practice based on what they learned in the course. In response to an opportunity to provide feedback about the didactic video, participants were very positive. For example, one participated felt positive about the fact that "doctors, patients, interactions, and communication specialists were involved," that the video was "very informative and information provided in manageable sections," and that ask-tell-ask and the components of informed consent were provided. They were less positive, however, about the fact that an entire patient-physician interaction was not provided, that some of the information was too basic, and that it was too long. Finally, in response to an opportunity to give general comments and suggestions, participants were generally positive ("Nice activity"; "very useful for clinicians not comfortable in this setting").

\section{Discussion}

Low accrual to clinical trials persists as a major challenge to testing new cancer treatments and translating them to tangible benefits for patients. Disproportionately low accrual among members of underrepresented minority groups further limits the generalizability of findings and represents a healthcare disparity, limiting access to promising new treatments for members of these groups.[1, 2] To address one important and understudied barrier to accrual, we developed and pilot-tested a physicianfocused communication training module as an intervention designed to improve how physicians discuss clinical trials with their patients. We developed the training module with stakeholder input and leveraged our archive of video-recorded naturally-occurring clinical trial discussions between oncologists and members of a diverse patient population to create training materials that were engaging and relevant to trainees' clinical practices.

Findings showed that, even among this experienced group of physicians, the module was acceptable and viewed favorably. More importantly, the module improved physican knowledge of discussing trials as well as some key attitudes and beliefs. After completing this training, physician were more likely to believe that clinical trials improved patient care. They also had more positive attitudes about the behavior of 
offering clinical trials to eligible patients, though this effect was only marginally statistically significant. The training did not have any significant influence on physicians' beliefs about the professional benefit of clinical trials or beliefs about resources physicians had available to them to support their engagement with clinical trials. These beliefs may be better targeted by interventions at the healthcare system level. For example, efforts focused on changing perceived norms around clinical trials, disseminating timely information about available trials and their eligibility criteria, and dedicating staff to support physcians in the consent and enrollment processes may complement the kind of provider-focused intervention described in this manuscript.

This module also did not have a significant influence on physicians' comfort in discussing clinical trials. However, this module was developed and tested in two NCl-designated comprehensive cancer centers with a strong research focus, high-functioning clinical trial infrastructure, and a diverse patient population. Most physicians who participated in this pilot study were very experienced at discussing trials in these settings, and their comfort in doing so was high even before the module. Nevertheless, we found encouraging improvements in several domains even among a group of physicians highly experienced in offering clinical trials. This training module may have even greater impact and usefulness among physicians less experienced in clinical trial discussions, such as primary care providers, community oncologists, or trainees. It may also be useful and easily adapted for providers such as nurses and physician assistants, who are valuable members of the healthcare team and who often discuss trials with patients and families.

\section{Limitations/Future Research}

Findings must be considered within the limitations of the study. First, in this pilot test, we evaluated the acceptability and preliminary effectiveness of this training module with a small sample of experienced physicians in two large, urban university-affiliated medical institutions. We propose that this sample provided a conservative test of the module's effectiveness. Still, future research should test the acceptability and effectiveness of this module among physicians with a greater variety of experience discussing trials, in community cancer hospitals with less of a focus on clinical research, and with nonphysician providers who are often critical to the process of enrolling patients to clinical trials. These efforts should utilize insights from dissemination and implementation science to inform adaptations to the existing intervention. Second, the outcomes were all self-reported by the participants and thus, we could not evaluate whether this training influenced physicians' communication behavior or increased patients' enrollment in trials. Observational methods such as analysis of video-recorded clinical interactions and other types of assessment of trial offers and patient decisions to participate, as planned in the larger parent study from which these data were derived,[25] could help determine to what extent and in what ways the module improves trial-related communication and accrual to trials in diverse patient populations.

Improving patient-physician communication about clinical trial research is critical to increasing patients' engagement in clinical trials. We designed a training module that described practical strategies 
physicians can use to meet the informational and relational needs of a diverse patient population. We reinforced this learning with opportunities for physicians to reflect on effective and ineffective communication strategies used by real physicians to address common challenges of clinical trial discussions. We enhanced the authenticity of this physician communication training by engaging stakeholders, including physicians, cancer survivors, and communication scientists, in the design and delivery of the training module. We also leveraged our archive of naturally-occurring patient-physician clinical trial discussions so that participants could view naturalistic examples of clinical trial discussions, rather than simulated discussions created for training purposes. This preliminary evidence supports the acceptability and effectiveness of the training module in enhancing physicians' knowledge, beliefs, and attitudes towards clinical trials. Our aim is that these improvements will translate to more frequent and higher-quality communication about clinical trials, especially with the diverse patient populations who stand to benefit most.

\section{Declarations}

Ethics approval and consent. Ethics approval was granted by the Institutional Review Boards of Wayne State University (IRB\# 066816B3E) and Johns Hopkins Medicine (IRB \#00108941)

Consent for Publication. Not applicable

Availability of Data and Materials. The data that support the findings of this study are available within the manuscript and from the corresponding author upon reasonable request.

Competing Interests: The authors declare they have no competing interests.

Funding. National Cancer Institute: R01 CA200718, P30 CA022453

\section{Authors' contributions:}

SE: Conception, design, data acquisition, analysis, interpretation, manuscript writing and approval of submitted version.

MM: Conception, design, analysis, interpretation, manuscript writing, and approval of submitted version.

NS: Conception, design, analysis, interpretation, manuscript writing, and approval of submitted version.

TFM: Conception, design, approval of submitted version.

TLA: Conception, design, approval of submitted version.

LAP: Conception, design, approval of submitted version.

EH: Conception, design, approval of submitted version

MAC: Conception, design, approval of submitted version 
DGL: Conception, design, approval of submitted version

LMH: Conception, design, data acquisition, analysis, interpretation, manuscript writing and approval of submitted version

\section{References}

1. Loree, J. M., S. Anand, A. Dasari, J. M. Unger, A. Gothwal, L. M. Ellis, G. Varadhachary, S. Kopetz, M. J. Overman, and K. Raghav. 2019. Disparity of Race Reporting and Representation in Clinical Trials Leading to Cancer Drug Approvals From 2008 to 2018. JAMA Oncol:e191870. doi:10.1001/jamaoncol.2019.1870.

2. Unger, J. M., D. L. Hershman, R. U. Osarogiagbon, A. Gothwal, S. Anand, A. Dasari, M. Overman, J. M. Loree, and K. Raghav. 2020. Representativeness of Black Patients in Cancer Clinical Trials Sponsored by the National Cancer Institute Compared With Pharmaceutical Companies. JNCl Cancer Spectr 4 (4):pkaa034. doi:10.1093/jncics/pkaa034.

3. Unger, J. M., R. Vaidya, D. L. Hershman, L. M. Minasian, and M. E. Fleury. 2019. Systematic Review and Meta-Analysis of the Magnitude of Structural, Clinical, and Physician and Patient Barriers to Cancer Clinical Trial Participation. J Natl Cancer Inst 111 (3):245-255. doi:10.1093/jnci/djy221.

4. Hamel, L. M., L. A. Penner, T. L. Albrecht, E. Heath, C. K. Gwede, and S. Eggly. 2016. Barriers to Clinical Trial Enrollment in Racial and Ethnic Minority Patients With Cancer. Cancer Control 23 (4):327-337.

5. Meropol, N. J., Y. N. Wong, T. Albrecht, S. Manne, S. M. Miller, A. L. Flamm, A. B. Benson, 3rd et al. . 2016. Randomized Trial of a Web-Based Intervention to Address Barriers to Clinical Trials. J Clin Oncol34 (5):469-478. doi:10.1200/JC0.2015.63.2257.

6. Jacobsen, P. B., K. J. Wells, C. D. Meade, G. P. Quinn, J. H. Lee, W. J. Fulp, J. E. Gray et al. . 2012. Effects of a brief multimedia psychoeducational intervention on the attitudes and interest of patients with cancer regarding clinical trial participation: a multicenter randomized controlled trial. J Clin Onco/30 (20):2516-2521. doi:10.1200/JC0.2011.39.5186.

7. Langford, A. T., S. T. Hawley, S. Stableford, J. L. Studts, and M. M. Byrne. 2020. Development of a Plain Language Decision Support Tool for Cancer Clinical Trials: Blending Health Literacy, Academic Research, and Minority Patient Perspectives. J Cancer Educ 35 (3):454-461. doi:10.1007/s13187019-1482-5.

8. Vuong, I., J. Wright, M. B. Nolan, A. Eggen, E. Bailey, R. Strickland, A. Traynor, and T. Downs. 2020. Overcoming Barriers: Evidence-Based Strategies to Increase Enrollment of Underrepresented Populations in Cancer Therapeutic Clinical Trials-a Narrative Review. J Cancer Educ 35 (5):841-849. doi:10.1007/s13187-019-01650-y.

9. Kim, E. S., S. S. Bruinooge, S. Roberts, G. Ison, N. U. Lin, L. Gore, T. S. Uldrick et al. . 2017. Broadening Eligibility Criteria to Make Clinical Trials More Representative: American Society of Clinical Oncology and Friends of Cancer Research Joint Research Statement. J Clin Oncol 35 (33):3737-3744. doi:10.1200/JC0.2017.73.7916. 
10. Albrecht, T. L., S. S. Eggly, M. E. Gleason, F. W. Harper, T. S. Foster, A. M. Peterson, H. Orom, L. A. Penner, and J. C. Ruckdeschel. 2008. Influence of clinical communication on patients' decision making on participation in clinical trials. J Clin Oncol 26 (16):2666-2673. doi:10.1200/JC0.2007.14.8114.

11. Wendler, D., R. Kington, J. Madans, G. Van Wye, H. Christ-Schmidt, L.A. Pratt, and Et al. 2006. Are racial and ethnic minorities less willing to participate in health research. PloS Med 3 (2):e19.

12. Eggly, S., T. L. Albrecht, F. W. Harper, T. Foster, M. M. Franks, and J. C. Ruckdeschel. 2008. Oncologists' recommendations of clinical trial participation to patients. Patient Educ Couns 70 (1):143-148. doi:10.1016/j.pec.2007.09.019.

13. Hamel, L. M., D. W. Dougherty, T. L. Albrecht, M. Wojda, A. Jordan, T. F. Moore, N. Senft et al. . 2020. Unpacking Trial Offers and Low Accrual Rates: A Qualitative Analysis of Clinic Visits With Physicians and Patients Potentially Eligible for a Prostate Cancer Clinical Trial. JCO Oncol Pract 16 (2):e124e131. doi:10.1200/JOP.19.00444.

14. Barton, E., and S. Eggly. 2009. Ethical or Unethical Persuasion?: The Rhetoric of Offers to Participate in Clinical Trials. Written Communication 26 (3):295-319. doi:10.1177/0741088309336936.

15. Shen, M. J., E. B. Peterson, R. Costas-Muniz, M. H. Hernandez, S. T. Jewell, K. Matsoukas, and C. L. Bylund. 2018. The Effects of Race and Racial Concordance on Patient-Physician Communication: A Systematic Review of the Literature. J Racial Ethn Health Disparities 5 (1):117-140. doi:10.1007/s40615-017-0350-4.

16. Eggly, S., E. Barton, A. Winckles, L. A. Penner, and T. L. Albrecht. 2015. A disparity of words: racial differences in oncologist-patient communication about clinical trials. Health Expect 18 (5):13161326. doi:10.1111/hex.12108.

17. Bylund, C. L., M. Michaels, E. S. Weiss, S. Patel, T. A. D'Agostino, M. C. Binz-Scharf, and D. McKee. 2020. The Impact of an Online Training Program About Cancer Clinical Trials on Primary Care Physicians' Knowledge, Attitudes and Beliefs, and Behavior. J Cancer Educ. doi:10.1007/s13187-02001731-3.

18. Brown, R. F., C. L. Bylund, Y. Li, S. Edgerson, and P. Butow. 2012. Testing the utility of a cancer clinical trial specific Question Prompt List (QPL-CT) during oncology consultations. Patient Educ Couns 88 (2):311-317. doi:10.1016/j.pec.2012.02.009.

19. Kissane, D. W., C. L. Bylund, S. C. Banerjee, P. A. Bialer, T. T. Levin, E. K. Maloney, and T. A. D'Agostino. 2012. Communication skills training for oncology professionals. J Clin Onco/ 30 (11):1242-1247. doi:10.1200/JCO.2011.39.6184.

20. Niranjan, S. J., R. W. Durant, J. A. Wenzel, E. D. Cook, M. N. Fouad, S. M. Vickers, B. R. Konety, S. B. Rutland, Z. R. Simoni, and M. Y. Martin. 2019. Training Needs of Clinical and Research Professionals to Optimize Minority Recruitment and Retention in Cancer Clinical Trials. J Cancer Educ 34 (1):26-34. doi:10.1007/s13187-017-1261-0.

21. Clever, S. L., R. A. Dudas, B. S. Solomon, H. C. Yeh, D. Levine, A. Bertram, M. Goldstein, N. Shilkofski, and J. Cofrancesco, Jr. 2011. Medical student and faculty perceptions of volunteer outpatients 
versus simulated patients in communication skills training. Acad Med 86 (11):1437-1442. doi:10.1097/ACM.0b013e3182305bc0.

22. Wendler, D., and C. Grady. 2008. What should research participants understand to understand they are participants in research? Bioethics 22 (4):203-208. doi:10.1111/j.1467-8519.2008.00632.x.

23. Kemp, E. C., M. R. Floyd, E. McCord-Duncan, and F. Lang. 2008. Patients prefer the method of "tell back-collaborative inquiry" to assess understanding of medical information. J Am Board Fam Med 21 (1):24-30. doi:10.3122/jabfm.2008.01.070093.

24. Kornburger, C., C. Gibson, S. Sadowski, K. Maletta, and C. Klingbeil. 2013. Using "teach-back" to promote a safe transition from hospital to home: an evidence-based approach to improving the discharge process. J Pediatr Nurs 28 (3):282-291. doi:10.1016/j.pedn.2012.10.007.

25. Eggly, S., L. M. Hamel, E. Heath, M. A. Manning, T. L. Albrecht, E. Barton, M. Wojda et al. . 2017. Partnering around cancer clinical trials (PACCT): study protocol for a randomized trial of a patient and physician communication intervention to increase minority accrual to prostate cancer clinical trials. BMC Cancer 17 (1):807. doi:10.1186/s12885-017-3804-5.

26. Somkin, C. P., A. Altschuler, L. Ackerson, A. M. Geiger, S. M. Greene, J. Mouchawar, J. Holup et al. . 2005. Organizational barriers to physician participation in cancer clinical trials. Am J Manag Care 11 (7):413-421.

\section{Table}




\begin{tabular}{|c|c|}
\hline & $\mathbf{N}(\%)$ \\
\hline Age & $5(26.3)$ \\
\hline $31-40$ & $5(26.3)$ \\
\hline $41-50$ & $8(42.1)$ \\
\hline $51-60$ & $1(5.3)$ \\
\hline \multicolumn{2}{|l|}{$\geq 61$} \\
\hline Gender & $15(79.8)$ \\
\hline Male & $4(21.1)$ \\
\hline \multicolumn{2}{|l|}{ Female } \\
\hline Race/Ethnicity & $11(57.9)$ \\
\hline White non-Hispanic & $2(10.5)$ \\
\hline Black non-Hispanic & $6(31.6)$ \\
\hline \multicolumn{2}{|l|}{ Asian } \\
\hline \multicolumn{2}{|l|}{ Site } \\
\hline Karmanos Cancer Institute (KCI) & $9(47.4)$ \\
\hline Sidney Kimmel Comprehensive Cancer Center (JHU) & $10(52.6)$ \\
\hline Medical Specialty & $9(47.4)$ \\
\hline Medical Oncology & $6(31.6)$ \\
\hline Radiation Oncology & $4(21.1)$ \\
\hline \multicolumn{2}{|l|}{ Urology } \\
\hline Years in Practice & $15(78.9)$ \\
\hline More than 5 years & $4(21.1)$ \\
\hline Less than 5 years & \\
\hline
\end{tabular}

Table 2: Pre- and Post-Test Correlations 


\begin{tabular}{|c|c|c|c|c|c|c|}
\hline & BEL: & BEL: & BEL: & ATT & KNOW & COMF \\
\hline & Patient & Profession & Resources & & & \\
\hline Beliefs: Patient Care & - & $.82^{* *}$ & .44 & $.76^{* *}$ & .04 & $.71^{* *}$ \\
\hline Beliefs: Profession & $.72^{\star \star}$ & - & $.60^{\star \star}$ & $.81^{\star \star}$ & .16 & $.83^{\star \star}$ \\
\hline Beliefs: Resources & $.48^{*}$ & $.70^{\star *}$ & - & $.47^{\star}$ & .31 & $.51^{*}$ \\
\hline Behavioral Attitudes & $.66^{\star \star}$ & $.85^{\star \star}$ & $.75^{\star \star}$ & - & .13 & $.69^{\star \star}$ \\
\hline Knowledge & .31 & $.61^{\star \star}$ & $.54^{*}$ & $.51^{*}$ & - & .29 \\
\hline Comfort & $.53^{*}$ & $.76^{* \star}$ & $.66^{* \star}$ & $.60^{\star \star}$ & $.57^{*}$ & - \\
\hline
\end{tabular}

Notes: Correlations below diagonal $=$ pre-training; above diagonal $=$ post-training. ${ }^{*}=p<.05,{ }^{*}=p<.01$ Table 3 RMANOVA results: Pre-Post Training Scores

\begin{tabular}{|c|c|c|c|c|c|c|c|c|}
\hline & \multicolumn{3}{|c|}{ Test of within-subject effect } & \multicolumn{2}{|c|}{ Pre-Training } & \multicolumn{2}{|c|}{ Post-Training } & \multirow[b]{2}{*}{$r_{\text {prepos }}$} \\
\hline & $F_{1,18}$ & $p$ & $\eta^{2}$ & $M$ & $S E$ & $M$ & $S E$ & \\
\hline Beliefs: Patient Care & 9.50 & 0.01 & 0.35 & 4.19 & 0.12 & 4.39 & 0.13 & $0.87^{\star \star}$ \\
\hline Beliefs: Profession & 0.14 & 0.72 & 0.01 & 4.54 & 0.13 & 4.53 & 0.13 & $0.93^{* \star}$ \\
\hline Beliefs: Resources & 0.19 & 0.67 & 0.01 & 3.99 & 0.17 & 3.95 & 0.19 & $0.86^{\star *}$ \\
\hline Behavioral Attitudes & 3.30 & 0.09 & 0.16 & 4.22 & 0.16 & 4.38 & 0.15 & $0.84^{\star \star}$ \\
\hline Knowledge & 12.64 & 0.00 & 0.41 & 8.37 & 0.38 & 9.79 & 0.30 & 0.32 \\
\hline Comfort & 2.12 & 0.16 & 0.11 & 4.53 & 0.14 & 4.42 & 0.16 & $0.89^{\star *}$ \\
\hline
\end{tabular}

$*=p<.05, * *=p<.01 . M=$ estimated marginal mean.

\section{Supplementary Files}

This is a list of supplementary files associated with this preprint. Click to download.

- CONSORTchecklist.doc 